\title{
Innovación en el aula de Historia: los efectos del Role-Playing en la educación secundaria
}

Innovation in the History classroom: the Role-Playing effects in high school

Sarah Elena Cuervo Sánchez $\left(^{*}\right)$

\section{Resumen}

La c omplejidad y la r elación c on diferentes procesos hi stóricos de l os c onceptos de primer orden hacen que su comprensión se torne ardua para los estudiantes. En este sentido, se ha desarrollado una experiencia en el aula centrada en medir el grado de aprendizaje alcanzado por el al umnado de 13 a ños en un c entro de ed ucación s ecundaria, s obre el concepto de primer orden panhelenismo. Dada la complejidad del concepto elegido, éste se ha dividido en 4 categorías subsidiarias. Para trabajar la mayoría de ellas se ha empleado el Role-Playing como actividad propicia para la comprensión de conceptos abstractos. Como instrumento de recogida de $\mathrm{d}$ atos s e ha $\mathrm{n}$ u tilizado formularios, I os c uales tras $\mathrm{s} u$ aná lisis $\mathrm{m}$ ediante I a taxonomía de SOLO $\mathrm{m}$ ostraron un a umento significativo en el gr ado $\mathrm{d}$ e aprendizaje o btenido $\mathrm{p}$ or $\mathrm{e}$ I estudiantado, quedando patente la relación entre las metodologías empleadas, las actividades realizadas y el grado de aprendizaje alcanzado.

Palabras Clave: Role-Playing, historia, c onceptos, di dáctica de la $\mathrm{H}$ istoria, apr endizaje, metodología activa, didáctica de las ciencias sociales.

\section{Abstract}

The $c$ omplexity and the r elationship b etween di fferent hi storical pr ocesses about $f$ irst-order concepts $m$ ake the s tudents' und erstanding of hi story $h$ arsh. B ased o $n t h i s$ situation in the classroom, has been carried out an experience for measuring the degree of learning achieved by 13-year-old students in a high school about the first-order concept: panhellenism. Given the complexity of the chosen concept, it was subdivided into four categories. To work with them and improve the understanding of abstract concepts, Role-Playing got applied as a suitable activity. Using forms as a dat a recollection instrument and analyzing them using the SOLO taxonomy showed a s ignificant increase in the d egree of I earning obt ained, s howing the relationship between the methodologies used, the activities executed, and the degree of learning achieved.

Key words: Role-Playing, history, concepts, History instruction, learning, active m ethodology, social sciences instruction.

\section{Introducción}

La asignatura de Historia se presenta con frecuencia con un carácter memorístico que no busca sino el almacenamiento de fechas, I ugares y datos aislados en la mente de los

* Historiadora independiente, sarah.cuervo@gmail.com, ORCID: 0000-0002-3550-0537

Cuervo Sánchez, S. E., (2021). Innovación en el aula de Historia: los efectos del Role-Playing en la educación secundaria. Clio. History and History Teaching, 47, 315-333. https://doi.org/10.26754/ojs clio/clio.2021475828 Recibido: 11/7/2021. Aceptado: 16/12/2021. 
estudiantes (Hernández Cardona, 2002). En esta línea, I os libros de texto se muestran grandes aliados de est e enf oque de la materia con contenidos tradicionales y co $\mathrm{n}$ un tratamiento somero de algunos de los procesos mas importantes de nuestra historia (Abril López y Cuenca Ló pez, 201 6). Esta i nvestigación contextualizada en esta r ealidad, busca medir el grado de aprendizaje adquirido por el estudiantado sobre conceptos de primer y segundo orden recurriendo a otras metodologías como el role-playing.

En est e se ntido, podr íamos definir el t érmino concepto co mo I a u nidad bási ca organizativa de co nocimiento est ructurado q ue se puede adq uirir (Barsalou, 1992), siendo básicos para organizar el mundo en el que vivimos y poder darle sentido. En didáctica de I a hi storia, son i gualmente nece sarios puesto que gracias a el los se construye una estructura básica que enseñará al estudiante a pensar históricamente (Rodríguez-Moneo y López, 2017). En un nivel más profundo de estudio, los conceptos se pueden s ubdividir en co nceptos de pr imer o rden, I os cuales llevan adscritos diferentes connotaciones, so $\mathrm{n}$ co mplejos y en $\mathrm{t}$ érminos históricos son el resultado último de diferentes procesos, y, por otro lado, los conceptos de se gundo orden, los cuales proporcionan las herramientas necesarias al estudiante para organizar la información disponible (Arteaga y Camargo, 2014).

El aprendizaje mediante conceptos consiste en crear redes de ideas que se forman a partir de conocimientos previos, conllevando irremediablemente a una reestructuración de los modelos preexistentes en el alumnado (Granados Sánchez y Pagès, 2011). Los conceptos de primer orden se caracterizan por ser abstractos y complejos, los cuales tan solo son explicados por los docentes de manera somera (Berti, 1994) y la mayoría de $\mathrm{I}$ as veces presentan $\mathrm{cl}$ aras dificultades al os estudiantes en c uanto a s $\mathrm{u}$ comprensión en se cundaria debido su desarrollo co gnitivo (Carretero y Montanero, 2008).

El presente estudio se centra en conceptos de contenidos curriculares propios de $1^{\circ}$ de $\mathrm{E}$ SO r eferentes a Historia A ntigua y por $t$ anto, $s$ egún I a t eoría del desa rrollo cognitivo de P iaget, los estudiantes de este curso, con 12-13 años, se encuentran en un estadio de oper aciones concretas, lo que supone que los educandos deberían ser capaces de realizar operaciones lógicas en la resolución de los problemas planteados (Rafael Linares, 2008).

De esta manera, el desarrollo cognitivo de nuestros estudiantes es algo fundamental a tener en cuenta tanto por la adaptación de contenidos necesaria como en lo relativo a la asi milación de co nceptos, pues to que las estructuras cognitivas no se encuentran aisladas al co nstruirlos. El est udiante no co nstruye o al tera su si stema co gnitivo durante e ste proceso, sino que al ex istir una i nteracción co gnitiva, és te experimenta una reestructuración sin perder su identidad (Gagliardi, 1986).

Por tanto, a través de este estudio se busca trabajar en torno al aprendizaje mediante un concepto de primer orden (el panhelenismo), enseñando a los discentes los pilares básicos para poder desa rrollar un pensamiento histórico, I o cu al co mporta una 
compleja exigencia cognitiva para los mismos (Carretero y Montanero, 2008; Salazar Jiménez, 2016) . Autores como V alverde B errocoso (2010), C arretero y M ontanero (2008) o Salueña Tolón (2016) han planteado que este tipo de aprendizaje no solo es favorecedor para el alumnado, sino que cuenta con un valor añadido ya que enseña al discente las herramientas necesarias para pensar históricamente.

En este caso, lo anteriormente explicado va de la mano de una serie de actividades planteadas en el aula, vease el Role-Playing como metodología que auna la teoría con la práctica. Esta actividad se planifica en torno a los objetivos de la investigación ya mencionados y se presenta como un juego para explicar las Guerras Médicas y sobre cómo los griegos superaron sus diferencias para unirse frente a un enemigo común. En este caso y sin contextualizar a los estudiantes previamente en las características del suceso, la clase se divide en dos bandos. A cada uno de ellos se les asigna un rol: Atenas o Esparta, cada polis con características asociadas. En este punto se plantea a los estudiantes la situación hipotética de una invasión por parte de la clase de en frente. Lo que conllevaría inexorablemente un inminente cambio en las normas del aula, un idioma diferente y el pago semanal de una cantidad de dinero a uno de los estudiantes de la otra clase.

Al reducir el juego a características con las que podían empatizar, la implicación de los estudiantes fue mayor así como su implicación. Fueron conscientes de las diferentes formas de gobierno que regían en cada polis para poder asumir su papel correctamente. De esta forma, tras una correcta dirección por parte del docente y una toma de decisiones de cada bando según su sistema de gobierno, ambos acordaron unir fuerzas ante la situación, dejando a un lado sus diferencias.

Esta actividad se enmarca en un proceso de Learning Cycle, el cual se ha demostrado tener una buena sinergia para el trabajo de conceptos (Brown y Abell, 2007; Marek, 2008; Pérez Ventura, 2017). La conjunción de actividades innovadoras y metodologías orientadas a est e fin favorecen l a co mprensión de i deas y conceptos complejos o abstractos. E $n$ est e se ntido, el R ole-Playing I lega a se $r$ una ex periencia r ealmente inmersiva par a I os educandos (Fuente A guilera, 2018; V alverde B errocoso, 2010 ). Además, se ha optado por trabajar mediante mapas conceptuales ya que ayudan a los estudiantes a $r$ elacionar diferentes conceptos, $f$ acilitando I a obt ención de una perspectiva general en el período histórico a trabajar, creando estructuras jerárquicas de i nformación que ay udan en I a co mprensión de I a m ateria (Kamaruddin y Idris, 2018).

\section{Metodología}

Tras insertar I a pr esente i nvestigación en un ca mpo de t rabajo t eórico, se t orna menester exponer en profundidad sus características. Se establece como pregunta de 
investigación la siguiente: "¿cuál es el grado de aprendizaje alcanzado por los estudiantes de $1^{\circ}$ de educación secundaria sobre el concepto de panhelenismo?" Y lleva asociados los siguientes objetivos generales:

- Establecer el grado de conocimiento previo de los estudiantes antes de la docencia par a fijar un punt o de par tida cl aro en $\mathrm{t}$ orno a l a cu al girará l a investigación.

- Analizar los resultados obtenidos en la presente investigación para medir el grado de conocimiento alcanzado, en los estudiantes de $1^{\circ}$ de educa ción secundaria de un concepto de primer orden, mediante el trabajo de aprendizaje basado en conceptos.

- Analizar la r elación ent re el g rado de co nocimiento obt enido en ca da categoría con las actividades realizadas durante la investigación, como forma de establecer cuáles de ellas son óptimas para el aprendizaje basado en conceptos.

La presente investigación se encu entra enm arcada co mo una de t ipo mixta, puesto que se procede a I a recolección y anál isis de datos cuantitativos y cualitativos, para finalmente realizar una interpretación resultante de toda la información en conjunto (Hernández et al ., 2017; P ereira Pérez, 2011). A sí co mo se $r$ de c arácter cu asi experimental, ya que la muestra sobre la que se va a trabajar no ha si do conformada exclusivamente par a l a i nvestigación, si no que viene dada por el ce ntro educativo donde se desarrolla la misma (Hernández et al., 2017). Es una muestra reducida por lo que el estudio se considera de carácter exploratorio.

A su vez esta investigación se ha desa rrollado en 2 fases. En una primera fase se ha llevado a ca bo una recogida de da tos, par a posteriormente en una s egunda fase realizar el análisis de los mismos. Este sistema mixto ha obtenido buenos resultados anteriormente y ha sido empleado a tal fin en anteriores experiencias en el aula (Pereira Pérez, 2011; Salueña Tolón, 2016).

Esta investigación gira en torno a una muestra compuesta por $\mathrm{N}=36$ estudiantes de 13 años de edad. El concepto el egido par a trabajar en el aula ha sido panhelenismo. Entendido este como "el programa político que pretende la unión de los griegos en una causa común que lleve a cabo la conquista del Imperio Persa para a través de esta acción, $\mathrm{r}$ esolver I os problemas endémicos de l a $\mathrm{H}$ élade a ni vel político, so cial y económico" (Antela-Bernárdez, 2007).

Este co ncepto se v uelve f undamental par a co mprender de una $\mathrm{m}$ anera integral Ia Antigua Grecia, puesto que en historia los conceptos de primer orden, son el resultado de pr ocesos de transformación que se fraguan dur ante déca das para finalmente terminar por manifestarse como un producto final, es lo que se conoce como visión de la hi storia baj o un pr isma de ca mbio y co ntinuidad (Paricio R oyo, 2018). Por la complejidad del concepto y las implicaciones de este, se ha di vidido en 4 categorías. 
En la conjunción de estas se encuentra el nacimiento mismo del concepto panhelenismo (Antela-Bernárdez, 2007):

- Categoría 1: misma cultura. Entendiendo po r el lo el mismo i dioma, r eligión, teatro, arquitectura, escultura, cultura material, deportiva y el resto de las producciones griegas que compartían unos determinados cánones.

- Categoría 2: sentimiento de libertad: Los griegos se veían a s í mismos como hombres libres.

- Categoría 3: enemigo común. El desencadenante final para el surgir de este sentimiento en el pueblo griego, fue la existencia de un enemigo común. Puesto que la amenaza persa en ciernes provocó la unión de todas las polis griegas bajo un mismo ejército que lucharía contra esta.

- Categoría 4: formas de gobierno. La pr incipal di ferencia ent re I os puebl os griegos, fueron las diferentes formas de gobierno de las polis. Sin embargo, a pesar de ello, reconocían que eran gobiernos creados por griegos libres.

De esta manera, la investigación inicia, en un a primera par te de la misma, con la recopilación de información pr evia a I a doce ncia, m ediante un cu estionario ar ticulado en $t$ orno a nuest ro concepto de pr imero rden y I as categorías expuestas anteriormente. Es to per mitirá establecer una muestra en cuanto al nivel cognitivo de partida del estudiantado.

Las preguntas hechas a los estudiantes fueron las siguientes:

1. Concepto $\mathbf{1}^{\mathrm{er}}$ orden: ¿Qué crees que significa panhelenismo?

2. Categoría 1: ¿Para qué crees que servía tener la misma lengua o religión en la Antigua Grecia?

3. Categoría 2: ¿Qué crees que significaba "ser libre" en la Antigua Grecia? ¿Qué importancia crees que tenía "ser libre"?

4. Categoría 3: ¿Quiénes crees que eran los enemigos de Grecia en aquella época? ¿Sabrías decir o imaginarte porque eran enemigos y no aliados?

5. Categoría 4: ¿Cómo crees que era el gobierno en la Antigua Grecia?

Posteriormente, se ha aso ciado una categoría a una actividad, la cual se ha diseñado exclusivamente a este propósito, haciendo uso de diferentes metodologías para favorecer la comprensión de cada una de ellas. No obstante, una misma actividad puede trabajar una o varias categorías de manera simultánea. Esto es lo que ocurre con la actividad de Role-Playing, mediante la cual se trabajan todas las categorías planteadas a l a vez que el co ncepto de pr imer or den se leccionado. Alguna de I as 
actividades desarrolladas mediante Lear ning $C$ ycle consistieron en el caso de la categoría 1 en el trabajo mediante imágenes, videos y texto de los antiguos deportes olímpicos y su relación con los actuales. Por lo que cada estudiante es el protagonista de su propio aprendizaje, creando sus propios puentes cognitivos.

Se establece así una relación entre las categorías, las preguntas planteadas en el cuestionario, $\mathrm{I}$ as actividades diseñadas para t rabajar cada una $\mathrm{d}$ e est as y l a metodología empleada en ellas (ver anexo: tabla 6).

En est e caso, se ha op tado por dividir el formulario final compuesto por las mismas preguntas mencionadas anteriormente, en 5 independientes. De esta manera, se dará el formulario final a los estudiantes tras la finalización de cada actividad dirigida que realicen. Esta parte de la investigación se ha diseñado de esta forma, puesto que la muestra es tá i nmersa en un pr ograma bi lingüe y por el lo se bu sca facilitar a lo s estudiantes la expresión de su s conocimientos. Mediante est a se gunda recogida de datos se busca comprobar la eficacia de las metodologías y actividades empleadas en cuanto al grado de aprendizaje adquirido por el estudiantado.

Posteriormente, se I levará a ca bo el anál isis de l os datos recolectados donde se determinará el g rado de co nocimiento adq uirido por el al umnado en cu anto al concepto de primer orden y las respectivas categorías. Este proceso se llevará a cabo mediante la taxonomía de SOLO. Este modelo nos muestra las distintas etapas que componen el aprendizaje, hast a un t otal de 4 di mensiones desde lo más superficial hasta lo más profundo y un nivel preestructural (Biggs y Tang, 2011).

Este modelo se ha puesto en práctica en estudios anteriores, con buenos resultados a la hor a de $\mathrm{m}$ edir el $\mathrm{g}$ rado de co nocimiento adq uirido (Hernández et al ., 2017 ; Rodríguez Gámez y Fernández Hernández, 2018; Salueña Tolón, 2016).

\section{Resultados}

La presente investigación se basa en el análisis de producciones escritas de los estudiantes, di stribuidas de I a siguiente manera: una pr egunta so bre el concepto de primer orden y $4 \mathrm{pr}$ eguntas sobre las categorías en las que hem os dividido est e concepto. Según se analizaron estas producciones se asignaron a una dimensión de la taxonomía de SOLO. En esta taxonomía se observan las diferentes dimensiones en cuanto al nivel de aprendizaje obtenido de menor a mayor. En las sucesivas tablas se asignará al ni vel pr eestructural el ni vel 0 en el $g$ rado de apr endizaje, a I $n$ ivel uniestructural, el nivel 1, al nivel multiestructural, el nivel 2, al nivel relacional, el nivel 3 y finalmente al nivel abstracta ampliada, el nivel 4.

\subsection{Concepto de $1^{\text {er }}$ orden. Panhelenismo.}


Se busca ba en e I est udiantado conocer el grado de co mprensión ace rca de l o q ué lleva arraigado el concepto Panhelenismo, en cuanto a la pertenencia a un hor izonte cultural común, independientemente de I as formas de gobierno que tenían Ias pol is vecinas. Primando por encima de todo una lengua, cultura y enemigo común que hizo que los griegos finalmente colaborasen, pero a su vez cada uno bajo el estandarte de su respectiva polis (ver anexo 2). Las dimensiones de la taxonomía de SOLO establecidas para esta cuestión fueron:

0. Preestructural: Respuesta equivocada o inexistente.

1. Uniestructural: Identifica la palabra como de origen griego.

2. Multiestructural: El estudiante describe el significado de la palabra panhelenismo.

3. Relacional: El estudiante es capaz de explicar las causas que engendraron este movimiento de unidad propio de la Antigua Grecia.

4. Abstracta ampliada: El estudiante reflexiona acerca de las implicaciones que este sentimiento de unidad política y cultural tuvo en la Antigua Grecia.

\begin{tabular}{|c|c|c|c|c|c|}
\hline Test & N.0 & N.1 & N.2 & N.3 & N.4 \\
\hline Inicial & 36 & 0 & 0 & 0 & 0 \\
\hline Final & 15 & 5 & 13 & 3 & 0 \\
\hline
\end{tabular}

Aquí podem os ver có mo en el t est final un $58,33 \%$ del estudiantado llegaron a comprender en alguna de sus dimensiones el concepto de panhelenismo. Sin embargo, hay un $41,67 \%$ que no llegó a comprenderlo.

\subsection{Categoría 1. Misma cultura.}

Mediante esta categoría esperaba que los estudiantes hubiesen comprendido que el hecho de co mpartir una $m$ isma cu ltura había jugado un papel muy importante en Ia unidad del pueblo griego. La música, la religión, la lengua, deportes, todo ello fue un factor cohesionador de la sociedad griega que permitió que el panhelenismo terminase por emerger. A mayores, se buscaba que los estudiantes extrapolasen esta situación con algunos de los nacionalismos que en la actualidad existen, todos ellos enmarcados precisamente en I a exi stencia de una cu Itura co mún de ntro de unas determinadas fronteras (ver anexo 3). Las dimensiones de l a taxonomía de S OLO adaptadas para esta cuestión fueron:

0. Preestructural: Respuesta equivocada o inexistente.

1. Uniestructural: El estudiante identifica que tener una misma lengua era útil.

2. Multiestructural: E I est udiante descr ibe I a ut ilidad de am bos conceptos como forma de comunicación o tener la misma religión como sinónimo de paz.

3. Relacional: El est udiante relaciona a mbos conceptos como u na forma de lograr una sociedad unificada.

4. Abstracta ampliada: El estudiante reflexiona sobre la importancia de ambos conceptos para lograr una sociedad unida y que como consecuencia intrínseca de la misma terminará generando un sentimiento de pertenencia y arraigo a un determinado territorio. 
En la tabla se observa cómo del test inicial al final hay un $22,22 \%$ de estudiantes que han abandonado el ni vel pr eestructural para denotar un mayor grado de aprendizaje. Pero sin duda, el dato más relevante en e ste

\begin{tabular}{|c|c|c|c|c|c|}
\hline Test & N.0 & N.1 & N.2 & N.3 & N.4 \\
\hline Inicial & 9 & 14 & 5 & 8 & 0 \\
\hline Final & 7 & 7 & 11 & 11 & 0 \\
\hline
\end{tabular}
gráfico es la diferencia en el nivel uniestructural, el cual del test inicial al test final, ha perdido un $50 \%$ de estudiantes ubicados en esa dimensión, puesto que han pasado a un nivel multiestructural. Este nivel ha visto incrementado el número de estudiantes en el test final en un $120 \%$.

\subsection{Categoría 2. Sentimiento de libertad.}

¿Son los estudiantes capaces de comprender la percepción que tenían los griegos de sí $\mathrm{m}$ ismos como puebl ol ibre, frente al os pueblos extranjeros que denominaban bárbaros? Y, en un nivel más profundo, de cómo esta percepción de sí mismos jugó un papel muy importante en la eclosión final del concepto panhelenismo (ver anexo 4). Las dimensiones de la taxonomía de SOLO adaptadas para esta cuestión fueron:

0. Preestructural: Respuesta equivocada o inexistente.

1. Uniestructural: El estudiante identifica una característica so bre el significado de "ser libre" o sobre la importancia de ser libre.

2. Multiestructural: El estudiante describe la importancia de "ser libre".

3. Relacional: $\mathrm{E}$ l est udiante relaciona am bos conceptos como algo que implícitamente es tán r elacionados con I a obt ención de der echos en I a A ntigua Grecia.

4. Abstracta ampliada: El estudiante reflexiona sobre la importancia que tenía ser un ciudadano libre en la Antigua Grecia, los derechos a los que podía optar por tener esa condición y es capaz de teorizar sobre la importancia que han supuesto los derechos humanos para la composición de nuestra sociedad.

\begin{tabular}{|c|c|c|c|c|c|}
\hline Test & N.0 & N.1 & N.2 & N.3 & N.4 \\
\hline Inicial & 26 & 1 & 5 & 4 & 0 \\
\hline Final & 6 & 1 & 8 & 21 & 0 \\
\hline
\end{tabular}

En la tabla se puede ver cómo un $76,92 \%$ de los estudiantes abandonaron el ni vel preestructural para terminar, la gran mayoría, en I a di mensión $r$ elacional, I a cu al ha $v$ isto aumentar su número en un $425 \%$.

\subsection{Categoría 3. Enemigo común.}

Mediante est a ca tegoría se busca ba que I os estudiantes comprendiesen la relación entre la existencia de un enemigo común, como lo fue el Imperio Persa en el siglo $\mathrm{V}$ 
a.C., con la necesidad indiscutible de las polis griegas de unirse dejando a un lado sus diferencias como único modo de supervivencia. Asimismo, que entendiesen que este fue el pun to de i nflexión q ue hizo q ue el resto de I as categorías mencionadas anteriormente desembocasen en el panhelenismo como sentimiento de pertenencia a un mismo horizonte cultural (véase anexo 5).

Las dimensiones de la taxonomía de SOLO adaptadas para esta cuestión fueron:

0. Preestructural: Respuesta equivocada o inexistente.

1. Uniestructural: El es tudiante identifica alguno de los enemigos que coexistieron con las polis griegas.

2. Multiestructural: El estudiante describe las razones por las que los enemigos de las polis griegas eran considerados como tales.

3. Relacional: El estudiante relaciona la existencia de un enemigo común con un sentimiento de unidad en la Antigua Grecia.

4. Abstracta ampliada: El estudiante reflexiona acerca del hecho que la existencia de un enemigo común en una sociedad (independientemente de la griega) conlleva inexorablemente un sentimiento de unidad de la misma.

El nivel pr eestructural ha su frido un notable descenso de estudiantes. $Y$ es que un $63,64 \%$ de los mismos, han abandonado esta dimensión para situarse en otras nuevas, siendo la dimensión multiestructural la que ha

\begin{tabular}{|c|c|c|c|c|c|}
\hline Test & N.0 & N.1 & N.2 & N.3 & N.4 \\
\hline Inicial & 33 & 0 & 3 & 0 & 0 \\
\hline Final & 12 & 5 & 19 & 0 & 0 \\
\hline
\end{tabular}
experimentado un mayor incremento con un $533,33 \%$ más de estudiantes.

\section{Categoría 4. Formas de gobierno.}

A pesar de lo anteriormente dicho, es necesario que el alumnado comprenda que las polis griegas eran ci udades-estado ( un co ncepto que ha si do trabajado en I as unidades precedentes) y como tales cada una es susceptible de tener diferentes formas de gobierno. Esto es cierto que provoca un punt o de desu nión dentro de la misma Grecia, sin embargo, el panhelenismo surge de igual manera, sobreponiéndose a las diferencias internas existentes en Grecia (ver anexo 6). Las dimensiones de la taxonomía de SOLO adaptadas para esta cuestión fueron:

0 . Preestructural: Respuesta equivocada o inexistente.

1. Uniestructural: El est udiante identifica al guna de I as formas de gobierno de I as polis griegas.

2. Multiestructural: El est udiante describe detalladamente las diferentes formas de gobierno de las polis griegas.

3. Relacional: El estudiante relaciona la existencia de los diferentes tipos de gobierno de las diferentes polis griegas con los tipos de gobiernos actuales. 
4. Abstracta a mpliada: El estudiante reflexiona acerca de Ia influencia que t uvo Ia civilización g riega en el dev enir pol ítico de I a so ciedad occi dental, $\mathrm{m}$ arcando claramente los cimientos de la democracia actual, así como la influencia de la oligarquía espartana en otras formas de gobierno posteriores como en la Alemania Nazi o la URRS.

\begin{tabular}{|c|c|c|c|c|c|}
\hline Test & N.0 & N.1 & N.2 & N.3 & N.4 \\
\hline Inicial & 33 & 3 & 0 & 0 & 0 \\
\hline Final & 7 & 5 & 18 & 6 & 0 \\
\hline
\end{tabular}

La tabla muestra un co ntraste m uy Ilamativo en el nivel preestructural, puesto que un $78,79 \%$ de los estudiantes han abandonado esta dimensión de I a t axonomía de S OLO para quedar finalmente representados en dimensiones como la multiestructural, donde I lama la at ención que en el primer test había 0 estudiantes y en el test final el $50 \%$ de ellos se encuentran en esta dimensión y $16,67 \%$ en la abstracta relacional.

\section{Discusión}

Mediante una lectura integral de los datos, es innegable que el grado de conocimiento de los estudiantes de la muestra ha aumentado. Sin embargo, es necesario ahondar aun $\mathrm{m}$ ás en el los y preguntarnos acerca de $\mathrm{I}$ as razones que han propiciado est $\mathrm{e}$ cambio, así co mo ace rca de cu áles han si do las actividades diseñadas para es ta investigación que han contribuido en mayor medida a favorecer este aprendizaje.

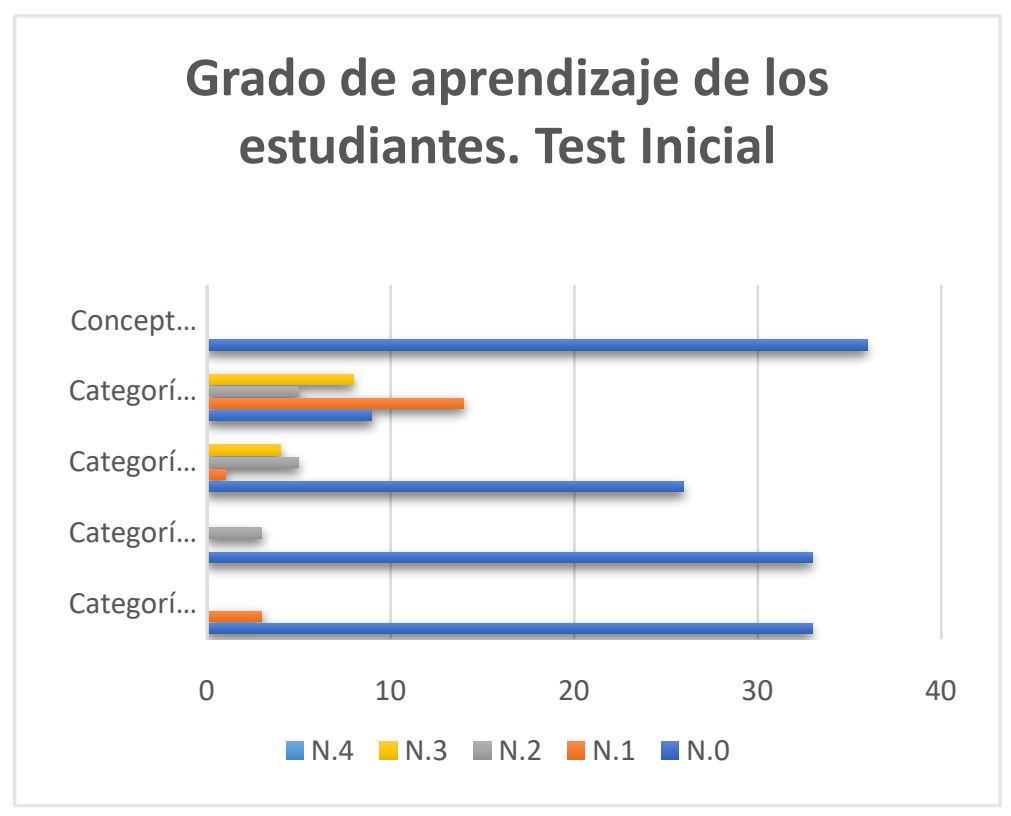




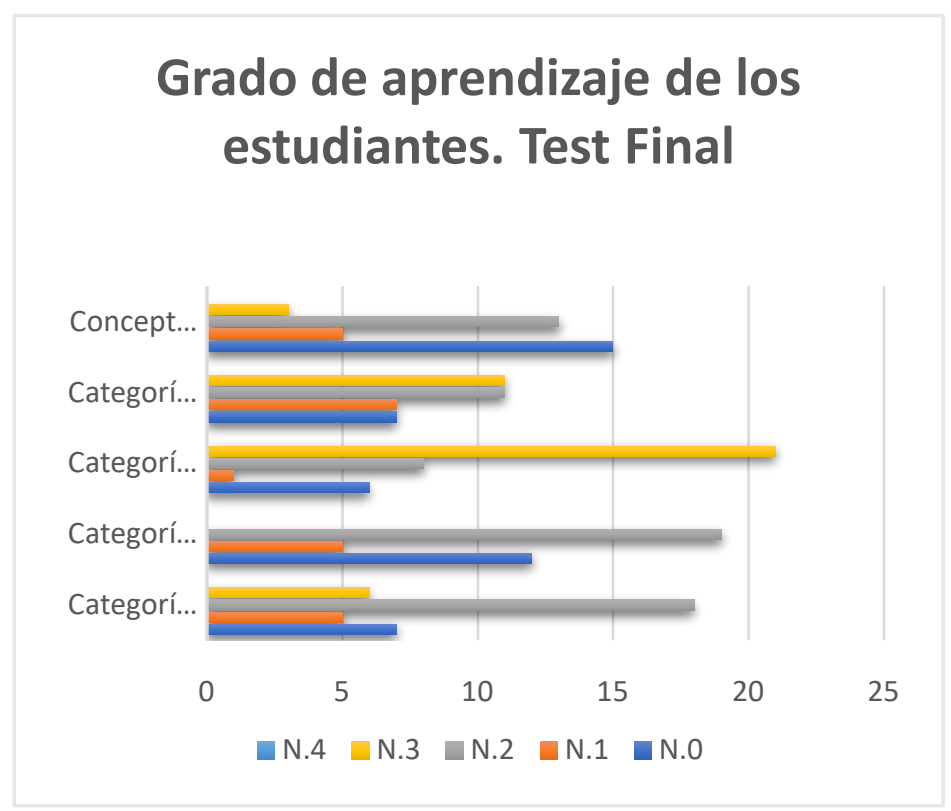

Es cierto que muchos estudiantes no han logrado establecer las relaciones cognitivas necesarias para ev aluar su apr endizaje den tro de al guna de I as dimensiones establecidas por la t axonomía de S OLO y por tanto se puede a firmar que se han quedado en un nivel preestructural. Este ha sido el caso del $41,67 \%$ de los estudiantes en el caso del concepto de primer orden, el $77,78 \%$ en el caso de la categoría 1 . El $23,08 \%$ en el caso de la categoría 2 . El $36,36 \%$ en el caso de la categoría 3 y el $21,21 \%$ en el caso de la categoría 4.

Y aq uellos estudiantes cuyo grado de aprendizaje ha cambiado de di mensión en I a taxonomía lo han hech o so lo hast a un ni vel multiestructural o r elacional, ninguno de ellos ha logrado llegar a una dimensión abstracta ampliada.

Si co mparamos los datos con ot ras investigaciones similares como la de Salueña Tolón (2016) vemos que en su caso el estudiantado tampoco ha logrado alcanzar una dimensión abstracta ampliada de I a taxonomía de S OLO, quedándose también en un nivel superficial de aprendizaje.

$Y$ es que, si bien es cierto que no todos los estudiantes han conseguido abandonar el nivel preestructural, es significativo el movimiento entre esta dimensión y las siguientes de la taxonomía de SOLO.

Es necesario se ñalar que en I a ca tegoría 1 el núm ero de e studiantes que han permanecido en el nivel preestructural es mayor puesto que en origen los estudiantes que se encontraban en este nivel era menor.

Como puede $v$ erse en el gr áfico 1, I lama I a at ención el grado de co nocimiento preexistente en la categoría 1 y 2 . Esto puede deberse a que los estudiantes simplemente extrapolan lo preguntado a su realidad actual. En el caso del uso de una lengua común, muchos de ellos, a tenor de lo que muestra el gráfico 1 , son capaces de establecer una relación entre una cultura similar y la existencia de periodos de paz, como un elemento de unión. 
Y en la categoría 2, se aprecia que hay un pequeño grupo de estudiantes que tienen conocimiento preexistente relacionado con el tema del se ntimiento de I ibertad. Esto puede ser der ivado de $q$ ue al vivir el los en un $m$ undo co $n$ todas las garantías de libertad asentadas, son capaces de extrapolar la pregunta a la realidad y apreciar la importancia de la libertad y su inexorable relación con la adquisición de derechos.

No obstante, es precisamente este conglomerado de cifras, presentado en el gráfico 1 y 2, el que debería hacernos reflexionar. $Y$ es que como se puede ver en la tabla del Anexo I, I a act ividad de Role-Playing se realiza enmarcada en una metodología de Learning Cycle y ha permitido trabajar de manera transversal las categorías 1, 2, 3 y 4. En las categorías 2, 3 y 4 se puede apreciar una mayor variación en cu anto al grado de aprendizaje alcanzado por los estudiantes. Puesto que un mayor número de el los han pasado de un aprendizaje preestructural a una dimensión uniestructural, multiestructural o relacional. El alumnado ha trabajado mediante esta actividad, 4 de las categorías planteadas, además del concepto de primer orden, sin ser ellos conscientes de haberlo hecho. Sin embargo, gracias a esta actividad el conocimiento adquirido po $r$ el los muestra no tables cambios como puede $v$ erse e $n$ los datos recogidos y lo que se puede apreciar en el gráfico 2. La actividad funcionó correctamente en a mbos grupos. Tras la misma, l a ac titud de I os estudiantes con respecto a los contenidos de la doce ncia ca mbió I igeramente, mostrando un $\mathrm{m}$ ayor compromiso por su parte.

\section{Conclusiones}

La asignatura de $\mathrm{H}$ istoria se viene estructurando desde hace déca das con frecuencia en exponer un relato único de hech os, sucesos personajes y fechas (Vicent et al., 2015). Sin embargo, es necesario abordar este hech o y plantear la didáctica de la Historia como un reto complejo que conlleva provocar la reacción nece saria en los estudiantes para desencadenar esa reestructuración del sistema cognitivo que en última i nstancia se $\mathrm{m}$ anifestará mediante la co mprensión de I os conceptos presentados por el docente. El papel que este juega se torna fundamental en este sentido, pues se co nvierte en el guía y m entor del est udiante en un camino que finalmente lo conducirá a tener una visión holística de la historia.

Tras el análisis de los datos recogidos y expresados en los gráficos 1 y 2 todo parece apuntar a I a ex istencia de una $r$ elación en tre I a $\mathrm{m}$ etodología, I as actividades empleadas y el grado de apr endizaje alcanzado por el estudiantado en I as diferentes categorías y concepto de primer orden.

De I a $\mathrm{m}$ isma $\mathrm{m}$ anera, queda pat ente q ue s e ha $\mathrm{r}$ espondido a I a pr egunta $\mathrm{d}$ e investigación, puesto que se ha constatado el grado de aprendizaje alcanzado por el alumnado que conforma la muestra. Asimismo, se han cumplido los objetivos planteados en la presente investigación ya que se ha logrado establecer un grado de 
conocimiento i nicial, para post eriormente mediante el anál isis y m edición de los mismos, establecer el grado de apr endizaje alcanzado por los estudiantes. EI tercer objetivo, relativo a analizar la relación entre las actividades desarrolladas en la presente investigación y el grado de conocimiento adquirido por el estudiante, también ha quedado patente. En este sentido, el Role-Playing se reafirma como una actividad que favorece la comprensión de conceptos complejos o abstractos (Fuente Aguilera, 2018; Valverde Berrocoso, 2010). En esta investigación se demuestra que esta actividad es beneficiosa par a el apr endizaje de conceptos, ay udando a c rear e sa reestructuración cognitiva en los estudiantes, aumentando notablemente el aprendizaje. Todo el lo enm arcado den tro de una $\mathrm{m}$ etodología Lear ning C ycle co mo pilar sobre el que sustentar un aprendizaje por descubrimiento y permitiendo el trabajo de varias categorías de manera simultánea.

No obstante, es necesario ser conscientes de que la muestra que se presenta en esta investigación es muy pequeña y por $t$ anto no es representativa $\mathrm{d}$ e I a realidad educacional, si no ua pr imera apr oximación o est udio ex ploratorio. Sin em bargo, la realización de este tipo de investigaciones es fundamental para conocer mejor a nuestro alumnado y, a su vez, conocernos mejor a nosotros mismos como docentes. Nos ayudan a elaborar una autocrítica y sobre todo nos ayudan a elegir la metodología y act ividades que $v$ amos a l levar a cabo en el aul $a$. $D$ espués de todo, se rá I a conjunción de ambas lo que se traducirá en un éxito o un fracaso a nivel educativo. En este sentido, la innovación y la investigación dentro del aula se presentan como elementos fundamentales para lograr mejoras en el ámbito educativo.

\section{Referencias}

Abril López, D., Cuenca López, J. M. (2016). Prehistoria y Arqueología en $1^{\circ}$ de ESO: análisis documental y propuesta didáctica para la explicación de la organización social pasada y ac tual. CLIO. History and History teaching, 42. http://clio.rediris.es/n42/articulos/abril2016.pdf

Antela-Bernardez, B. (2007). Hegemonía y Panhelenismo: Conceptos Políticos en tiempos de Filipo y Alejandro. Dialogues D Histoire Ancienne, 33(2), 69 -89. https://www.persee.fr/doc/dha 0755-7256 2007 num 3323041

Arteaga, B ., C amargo, S . (2014). E ducación h istórica: una pr opuesta par a e I desarrollo de I pensamiento histórico en el plan de estudios de 2012 para la formación de maestros de Educación Básica. Tempo e Argumento, 6(13), 110-140.

Barsalou, L. W. (1992). Frames, concepts, and conceptual fields. New Essays in Semantic and Lexical O rganization. E n Frames, Concepts, and Conceptual Fields (pp. 21 -74). Lawrence Erlbaum Associates.

Berti, A. E. ( 1994). C hildren's u nderstanding of the c oncept of the s tate. En Cognitive and Intructional Processes in History and Social Sciences (pp. 49-77). Lawrence Earlbaum Associates. 
Biggs, J., Tang, C. (2011). Knowledge and understanding. En Teaching for Quality Learning at University (pp. 8 1-95). O pen University $P$ ress

https://cetl.ppu.edu/sites/default/files/publications/-John Biggs and Catherine TangTeaching for Quali-BookFiorg-.pdf

Brown, P. L., Abell, S. K. (2007). Examining the Learning Cycle. Perspectives, 58-59.

Carretero, M. , y Montanero, M. ( 2008). E nseñanza y apr endizaje de I a $\mathrm{H}$ istoria: as pectos cognitivos y culturales. Cultura y Educación, 20(2), 133-142.

Fuente Aguilera, A. (2018). La simulación y el role playing como metodologías activas en el aula de Historia: "Corresponsales de guerra": una propuesta didáctica para abordar la II Guerra Mundial [Trabajo de fin de máster, Universidad de Burgos]. Riubu-e A rchivo. http://hdl.handle.net/10259/5109

Gagliardi, R . ( 1986). Los c onceptos estructurales en el apr endizaje p or i nvestigación. Enseñanza de las ciencias: revista de investigación y experiencias didácticas, 4(1), 3035. https://www.raco.cat/index.php/Ensenanza/article/view/50857

Granados Sánchez, J., Pagès, J. (2011). Las unidades didácticas de conocimiento del medio social y c ultural. E n Didáctica del Conocimiento del Medio Social y Cultural en la Educación Primaria (pp. 145-161). Síntesis.

Hernández Cardona, F. X. (2002). Didáctica de las ciencias sociales, geografía e historia (Vol. 169). Graó Educación.

Hernández, R., Fernández, C., Baptista, P. (2017). Metodología de la investigación (6. ${ }^{\circledR}$ ed.). McGraw-Hill Education.

Kamaruddin, N., I dris, S. (2018). The Effectiveness Use of Mind Maps in Learning 4th years History Textbooks. Journal of Educational System, 2(4), 6-14.

Marek, E. A. (2008). Why the Learning Cycle? Journal of Elementary Science Education, 20(3), 63-69. https://doi.org/10.1007/BF03174709

Paricio Royo, J. (2018). E I v alor de I a hi storia. E studio de alternativas curriculares en Secundaria (1): una visión integrada de las transformaciones (cambio/continuidad) que conducen y modelan el presente (conciencia histórica). Clío: History and History Teaching, 44. http://clio.rediris.es/n44/articulos/14Paricio2018.pdf

Pereira Pérez, Z. (2011). Los diseños de método mixto en la investigación en educación: Una experiencia c oncreta. Revista Electrónica Educare, 15(1), 15 -29. https://doi.org/10.15359/ree.15-1.2

Pérez Ventura, J. (2017). El aprendizaje por conceptos y el método learning cycle. Resultados de una ex periencia i nnovadora. Ventura Educación. https://vaventura.com/wpcontent/uploads/2017/05/T1052017-Aprendizaje-conceptos.pdf

Rafael Linares, A. (2008). Desarrollo Cognitivo: Las teorías de Piaget y Vygotsky. Universidad Autónoma de Barcelona http://www.paidopsiquiatria.cat/FILES/TEORIAS DESARROLLO COGNITIVO 0.PDF

Rodríguez Gámez, W. H., F ernández Hernández, F . (2018). U so de la taxonomía S OLO en situaciones de muestreo: un ejemplo de ap licación. Números: revista didáctica de matemáticas, 98, 105-116. 
Rodríguez-Moneo, M., Lopez, C. (2017). Concept acquisition and conceptual change in History. Palgrave Handbook of Research in Historical Culture and Education, (pp. 46 9-490). Palgrave. https://doi.org/10.1057/978-1-137-52908-4 25

Salazar Jiménez, R. A. (2016). La construcción del discurso histórico en estudiantes de ESO: El aula como laboratorio. [Tesis doctoral, Universitat de Barcelona]. Dipòsit Digital de la Universitat de Barcelona. http://hdl.handle.net/2445/100712

Salueña T olón, E . ( 2016). E studio s obre el a prendizaje por c onceptos e $\mathrm{n}$ al umnos de Educación Secundaria en las Ciencias Sociales. Experiencia en un centro edu cativo. CLIO. History and History teaching, 42.

Valverde B errocoso, J . ( 2010). A prendizaje de I a H istoria y S imulación Educativa. Tejuelo: didáctica de la Lengua y la Literatura, 9, 83-99.

Vicent, N ., R ivero, P., F eliu, M. (2015). Arqueología y t ecnologías d igitales en E ducación Patrimonial. Educatio siglo XXI: Revista de la Facultad de Educación, 33(1), 83-102.

Publicado bajo licencia internacional Creative Commons Attribution-NonCommercial-ShareAlike4. $\phi$ (CC-BY-NC-SA 4.0). Se permite copiar, usar, distribuir, transmitir y exhibir públicamente, siempre que: i) se reconozca la autoría y la fuente original de publicación (revista, ed itorial y URL del trabajo); ii) no se utilice con fines comerciales; iii) se mencione la existencia y especificaciones de esta licencia. 


\section{ANEXO 1: RELACIÓN ENTRE PREGUNTA DEL CUESTIONARIO, ACTIVIDAD REALIZADA Y METODOLOGÍA EMPLEADA}
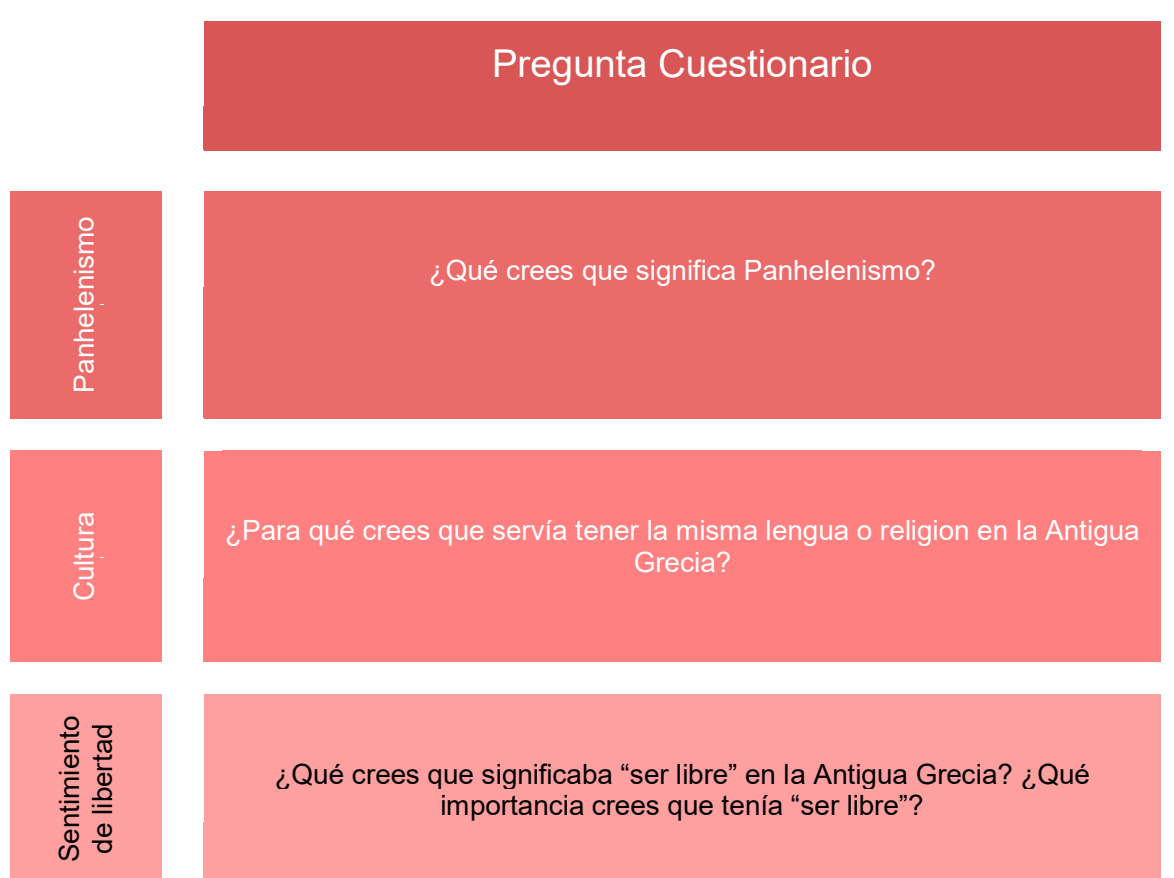

¿Qué crees que significaba "ser libre" en la Antigua Grecia? ¿Qué importancia crees que tenía "ser libre"?

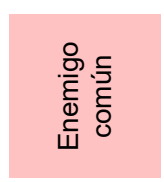
¿Quiénes crees que eran los enemigos de Grecia en aquella época? ¿Sabrías decir o imaginarte por qué eran enemigos y no aliados?

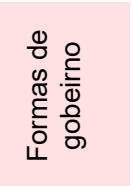

¿Cómo crees que era el gobierno en la Antigua Grecia?
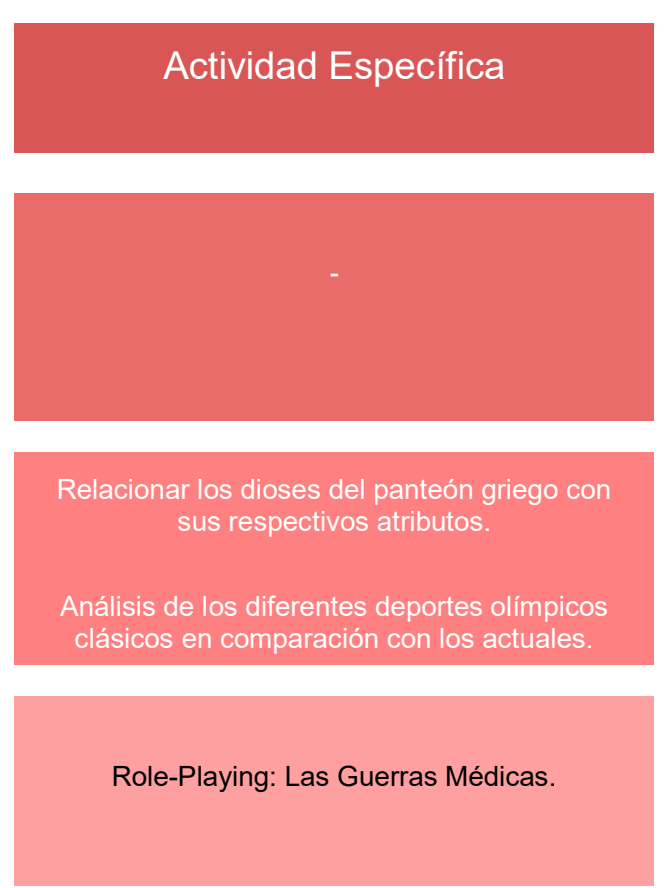

Role-Playing: Las Guerras Médicas.

Role-Playing: Las Guerras Médicas.
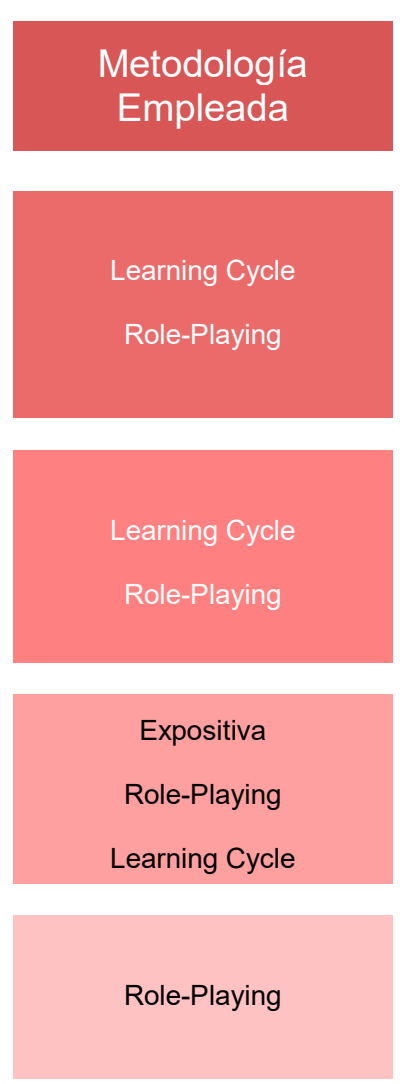

Role-Playing

Aprendizaje significativo 
ANEXO 2:

Ejemplos de $r$ espuesta por par te de I alumnado en torno a I c oncepto de primer orden en el test final:

0. Preestructural:

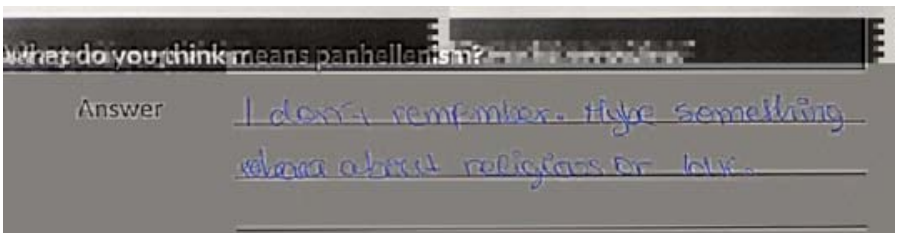

1. Uniestructural:

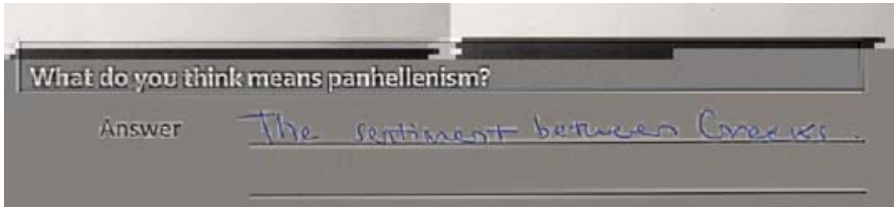

2. Multiestructural:

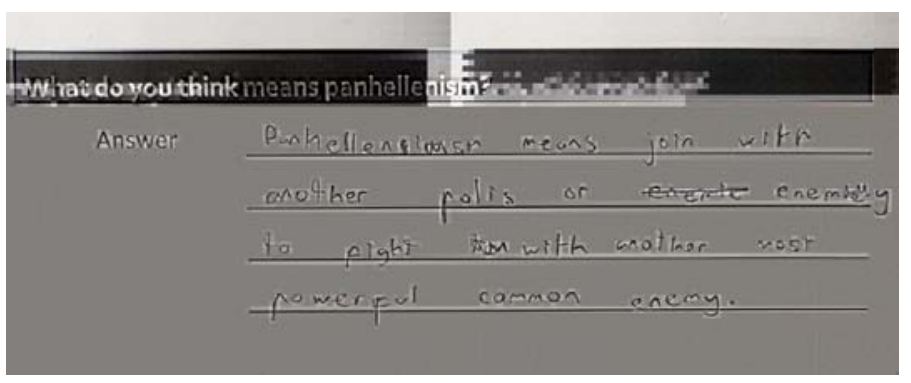

3. Relacional:

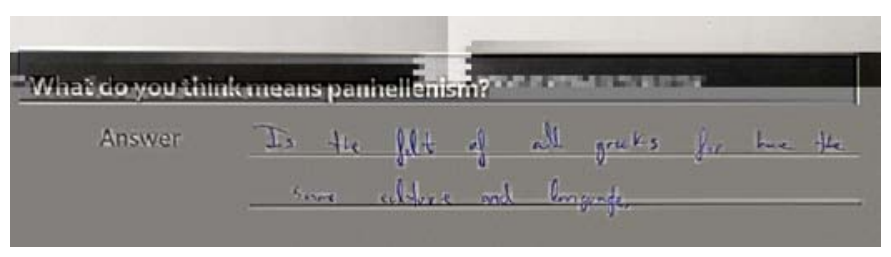

4. Abstracta ampliada:
ANEXO 3:

Ejemplos de $r$ espuesta por par te de I alumnado e $\mathrm{n}$ t orno a la c ategoría 1 en el test final:

\section{Preestructural:}

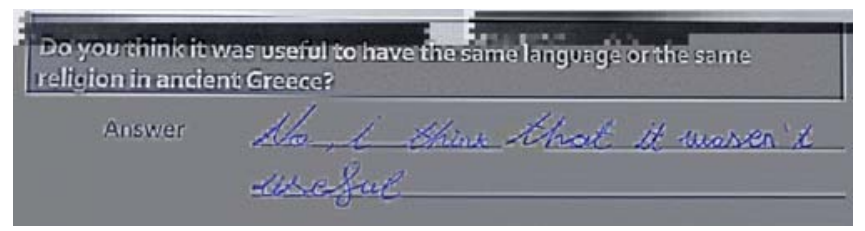

1. Uniestructural:

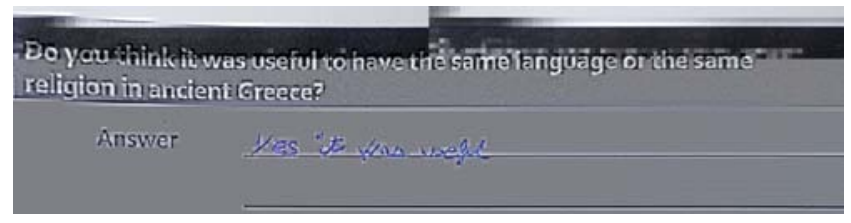

2. Multiestructural:

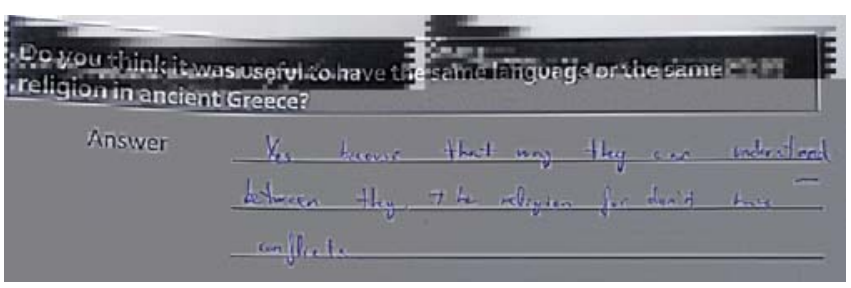

3. Relacional:

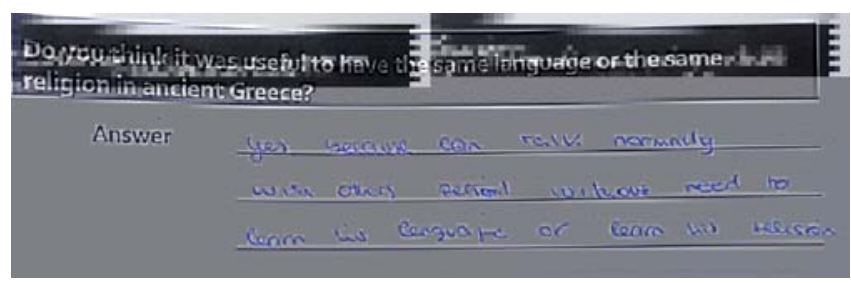

4. Abstracta ampliada: 
ANEXO 4:

Ejemplos de $r$ espuesta por par te de I alumnado e $\mathrm{n}$ t orno a la c ategoría 2 en el test final:

0. Preestructural:

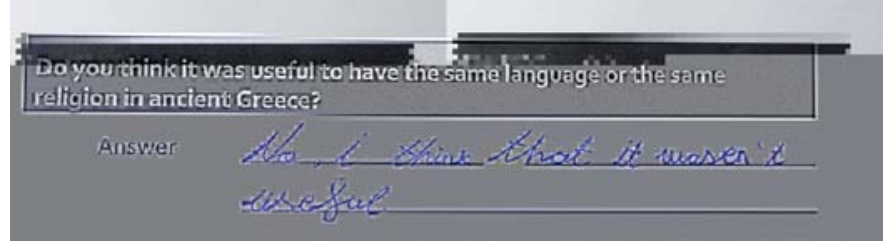

1. Uniestructural:

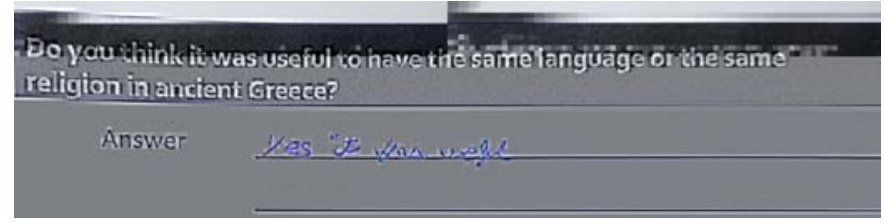

2. Multiestructural:

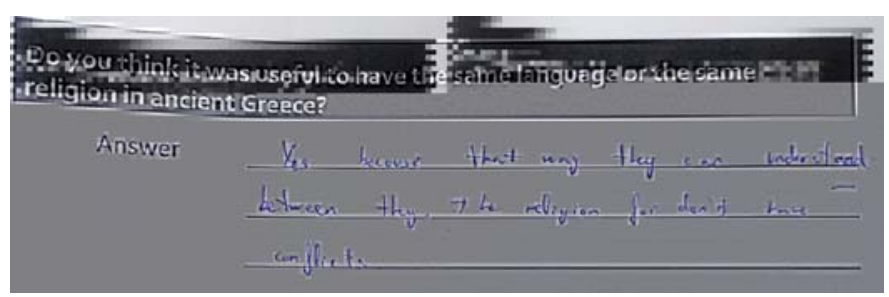

3. Relacional:

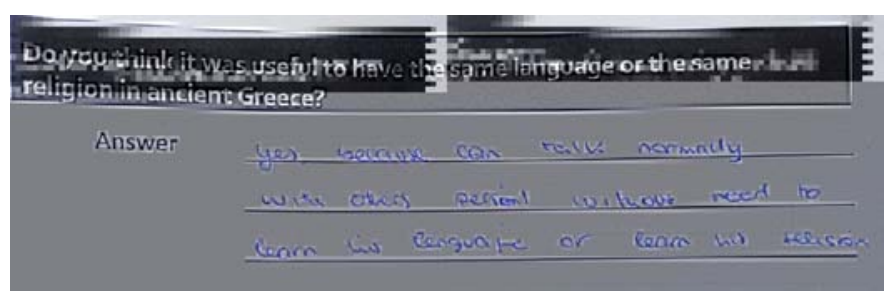

4. Abstracta ampliada:

\section{ANEXO 5:}

Ejemplos de $r$ espuesta por par te de I alumnado e $\mathrm{n}$ t orno a la c ategoría 3 en el test final:

0. Preestructural:

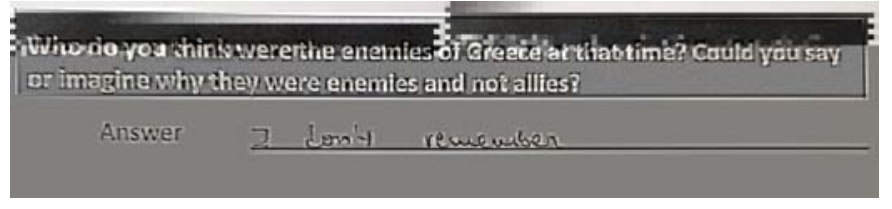

1. Uniestructural:

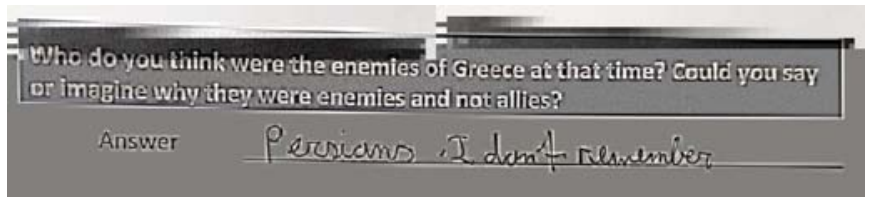

2. Multiestructural:

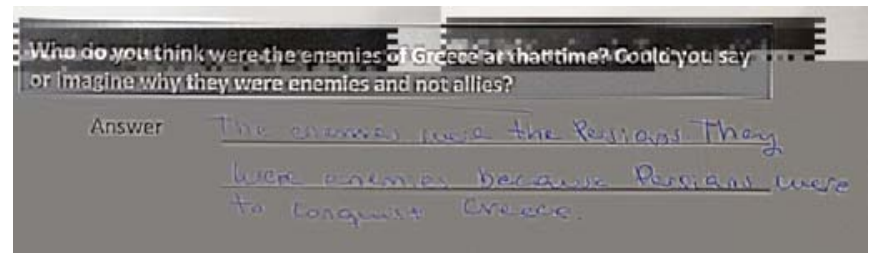

3. Relacional:

4. Abstracta ampliada: 
ANEXO 6:

Ejemplos de $r$ espuesta por par te de I alumnado e $n$ t orno a la c ategoría 4 en el test final:

0. Preestructural:

\section{How do you think the government was like in ancient Greece?}

Answer $-\operatorname{incitis}$

1. Uniestructural:

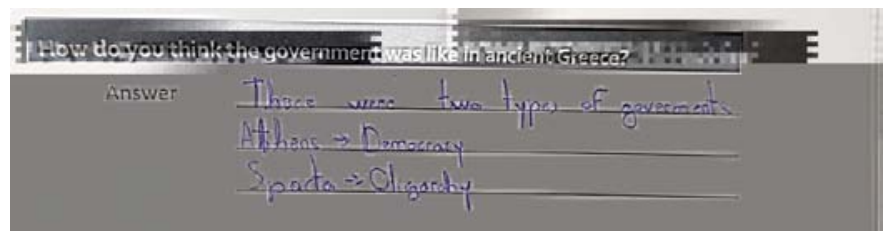

2. Multiestructural:

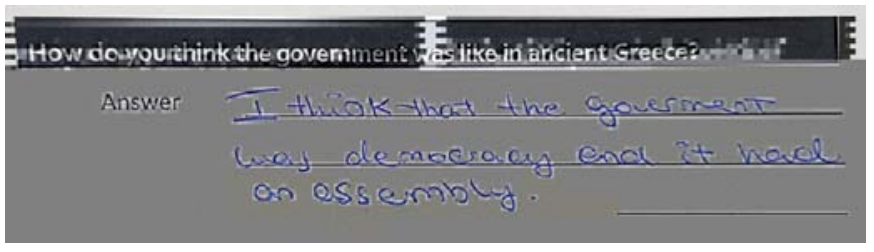

3. Relacional:

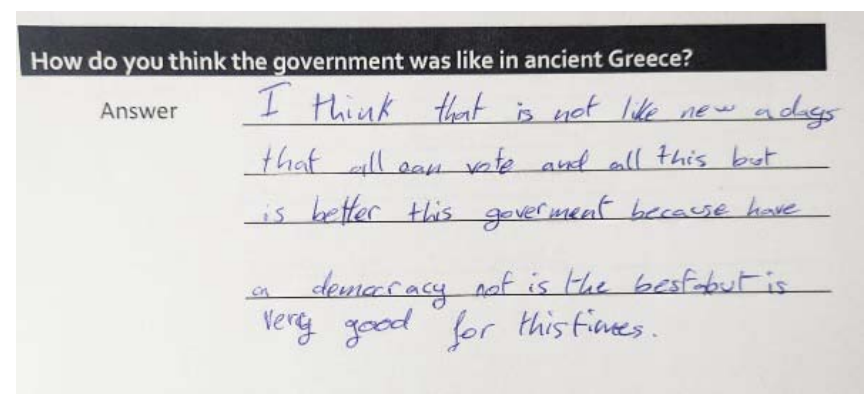

4. Abstracta ampliada: 